\title{
La expresión de sentimientos como promotor de bienestar subjetivo en una paciente de cáncer
}

The expression of feelings as a promoter of subjective well-being in a cancer patient A expressão de sentimentos como promotor do bem-estar subjetivo em um paciente com câncer

\begin{abstract}
Ana Karen Osornio García
Universidad Michoacana de San Nicolás de Hidalgo, Facultad de Psicología, México psic.karenosornio@gmail.com https://orcid.org/0000-0001-5802-5962

Belem Medina Pacheco

Universidad Michoacana de San Nicolás de Hidalgo, Facultad de Psicología, México belemed@yahoo.es https://orcid.org/0000-0001-9630-7274 María Inés Gómez del Campo del Paso Universidad Michoacana de San Nicolás de Hidalgo, Facultad de Psicología, México ines.paso@umich.mx https://orcid.org/0000-0001-9529-4635
\end{abstract}

\section{Resumen}

La presente investigación se basó en la expresión de sentimientos como promotor de bienestar subjetivo en una paciente de cáncer, en el Hospital de Oncología en la ciudad de Morelia, Michoacán, México. La investigación estuvo enmarcada en el estudio cualitativo y se utilizó el método fenomenológico mediante la estrategia de un estudio de caso. Para ello, se emplearon una serie de técnicas e instrumentos de recolección de datos, específicamente la entrevista a profundidad, la narrativa y la observación directa. El objetivo fue analizar la 
manera en que la expresión de sentimientos con respecto a su enfermedad, en una paciente con cáncer, promueve su bienestar subjetivo a través de una intervención psicocorporal.

De esta manera se pudo concluir que los sentimientos presentes en la paciente respecto a la enfermedad fueron el enojo, la tristeza y el miedo. Se encontró que la represión es el manejo que la participante le da a sus emociones, ya que al sufrir una enfermedad crónicodegenerativa se presentan emociones del pasado. La intervención le dio la oportunidad a la participante para hablar de los sentimientos respecto a su enfermedad. Al permitirse expresar sus sentimientos pudo encarar de mejor modo la enfermedad, e incluso surgieron sentimientos positivos relacionados con la satisfacción con su vida y las ganas de superar el cáncer.

Palabras clave: bienestar subjetivo, paciente de cáncer, sentimientos.

\section{Abstract}

The present investigation was based on the expression of feelings as a promoter of subjective well-being in a cancer patient, at the Oncology Hospital in the city of Morelia, Michoacán, Mexico. The research was framed with a type of qualitative study and the phenomenological method was used through the strategy of a case study. For the case study, a series of data collection techniques and instruments were used, specifically the in-depth interview, narrative and direct observation. The objective under which we worked was to analyze the way in which the expression of feelings regarding her illness, in a patient with cancer, promotes her subjective well-being, through a psycho-corporeal intervention.

In this way, it was possible to conclude that the feelings present in the patient regarding the disease were anger, sadness and fear. It was found that repression is the management that the participant gives to her emotions, since when she is in a chronic degenerative disease, emotions from the past are presented. The intervention gave the participant the opportunity to talk about her feelings regarding her illness, to express and identify them. Allowing yourself to express your feelings has given you the opportunity to function better in the face of the disease, emerging positive feelings, expressing satisfaction with your life, and wanting to overcome cancer.

Keywords: Subjective well-being, cancer patient, Feelings. 


\section{Resumo}

A presente investigação se baseou na expressão de sentimentos como promotores de bemestar subjetivo em um paciente com câncer, no Hospital de Oncologia da cidade de Morelia, Michoacán, México. A pesquisa enquadrou-se no estudo qualitativo e utilizou-se o método fenomenológico por meio da estratégia de um estudo de caso. Para tanto, foi utilizada uma série de técnicas e instrumentos de coleta de dados, especificamente a entrevista em profundidade, narrativa e observação direta. O objetivo foi analisar como a expressão de sentimentos em relação à sua doença, em uma paciente com câncer, promove seu bem-estar subjetivo por meio de uma intervenção psico-corporal.

Desse modo, foi possível concluir que os sentimentos presentes no paciente em relação à doença foram raiva, tristeza e medo. Constatou-se que a repressão é o manejo que a participante dá às suas emoções, visto que, ao sofrer uma doença crônico-degenerativa, são apresentadas as emoções do passado. A intervenção deu à participante a oportunidade de falar sobre seus sentimentos em relação à sua doença. Ao se permitir expressar seus sentimentos, conseguiu enfrentar melhor a doença, surgindo até sentimentos positivos relacionados à satisfação com a vida e ao desejo de superar o câncer.

Palavras-chave: bem-estar subjetivo, paciente com câncer, sentimentos.

Fecha Recepción: Enero 2021

Fecha Aceptación: Julio 2021

\section{Introducción}

El cáncer es una de las principales enfermedades de nuestro tiempo debido a su elevada prevalencia, morbilidad y mortalidad - Insa (2010) citado por Palacios-Espinosa, González y Zani (2015)— _ Las personas diagnosticadas con cáncer se ven afectadas, además de la enfermedad, por la forma como son tratadas por la sociedad, lo que dificulta su adecuada expresión emocional. Por ello, realizar este trabajo tiene gran importancia, ya que se pretende contribuir al bienestar subjetivo de las personas. Cabe señalar que esta población tiene una representación negativa de la enfermedad, ya que la consideran mortal. Esto hace que se asocie con emociones negativas muy intensas (tristeza, miedo, dolor y sufrimiento) y con diálogos internos de derrota y desamparo (Palacios-Espinosa et al., 2015). En otras palabras, todos los efectos psicológicos que sufren los pacientes al enfrentarse con esta enfermedad se originan en sus valoraciones; es decir, si el paciente lo valora como un trauma, terminado el tratamiento, puede tener un síntoma del trastorno de estrés postraumático debido a las 
amenazas o cambios que sufrió en todo el proceso (Pontaza, 26 de octubre de 2017). Adaptarse a vivir con una enfermedad de esta naturaleza exige tiempo, paciencia, apoyo e interés por participar en el cuidado de la propia salud. Por eso, entender lo que ocurre y participar activamente en el cuidado de la propia salud ayuda a afrontar los nuevos retos que van apareciendo (Correa, 2017).

Ahora bien, los sentimientos pertenecen a un proceso natural y surgen de una emoción respecto a una situación vivida. En este contexto, el bienestar subjetivo es un tema que actualmente es estudiado por la psicología positiva, y se refiere a la evaluación que la persona hace desde su perspectiva de vida (Verdugo et al., 2013).

Por ello, el objetivo general de esta investigación fue analizar la manera en que la expresión de sentimientos con respecto a su enfermedad promueve su bienestar subjetivo en una paciente con cáncer. Los objetivos específicos son los siguientes:

- Identificar los sentimientos presentes sobre su enfermedad en una paciente con cáncer a través de una intervención con técnicas psicocorporales.

- Analizar el manejo que da a sus emociones la participante de la investigación durante una intervención con técnicas psicocorporales.

- Analizar la influencia que genera la expresión de sentimientos en el bienestar subjetivo de la participante.

\section{Emociones y sentimientos}

Las emociones son el resultado de la evaluación de una situación por parte del organismo. Las emociones y todas las reacciones corporales asociadas a ellas sirven de fundamento para los mecanismos básicos de la regulación de la vida. Estas se constituyen por reacciones simples y son espontáneas (Muñoz, 2016).

Pallarés (2013) define las emociones como agitaciones o estados de ánimo producidos por ideas, recuerdos, apetitos y deseos. Son impulsos irracionales, adaptaciones a los cambios externos o internos y consecuencias de ideas mantenidas que se ejecutan en determinado momento. Las emociones se representan en el teatro del cuerpo, mientras que los sentimientos lo hacen en el teatro de la mente. Las emociones son reacciones diseñadas para ayudarnos a superar determinados cambios externos que pueden afectar nuestra integridad. Muñoz (2016) menciona algunas características más importantes de las emociones: 
- Preceden al sentimiento y dependen de las sensaciones y percepciones.

- Suelen ser intensas, pero de corta duración.

- Son el resultado de la evaluación de la situación por parte del organismo.

- Están al servicio de la supervivencia.

- Pueden ser observadas por otros a través de acciones o movimientos que pueden verse en conductas específicas de tipo no verbal: en la cara, la voz, los gestos, los cambios hormonales, etc.

Duque y Vico (2012) refieren algunos componentes de las emociones:

- Cumplen diversas funciones, entre las cuales se encuentran la preparación para la acción, la conformación del comportamiento futuro y la regulación de la interacción social.

- Las emociones son sentimientos que suelen tener un componente fisiológico y cognitivo.

- Las emociones son intensas y van acompañadas de diversos cambios fisiológicos; entre ellos, aceleración de ritmos respiratorios y cardiacos, resequedad de la boca, aumento de la sudoración y en ocasiones piloerección.

La emoción da a la vida intensidad y significado (Ramos Rocha, 2004). Es una cualidad de la experiencia y una cualidad expresiva de la acción. Por eso, se recomienda ser libre para experimentar la gama completa de emociones debido a que ofrece un enriquecimiento en la calidad de vida. En tal sentido, resulta vital que los sentimientos de la persona sean auténticos, pues las emociones actuadas o pretendidas falsean las relaciones con los demás. Tal inautenticidad determina la enfermedad y distorsiona el crecimiento personal.

La emoción no dura mucho tiempo (de 15 a 20 minutos), pues no podríamos soportar su intensidad. Por eso, tenemos un límite natural y espontáneo que hace disminuir su ímpetu. En este sentido, vale señalar que tenemos emociones primarias, las cuales son innatas y están a nuestra disposición para satisfacer las necesidades principales y para funcionar como instinto de supervivencia. Las emociones básicas son el miedo, el afecto, la tristeza, el enojo y la alegría. 
- Miedo: El miedo es la reacción que surge ante la percepción de una amenaza. Este nos hace buscar protección y normalmente se expresa en forma de huida. Implica una sensación de desagrado, aunque al mismo tiempo nos hace estar alertas. Si no es experimentada adecuadamente, la persona no lograría cuidar de sí misma en momentos de peligro.

- Afecto: El afecto tiene que ver con la atracción hacia otro o hacia algo que puede satisfacer alguna necesidad. Es la base para generar vínculos con el medio, aunque implica una sensación de agrado. Si no se experimenta adecuadamente, seríamos incapaces de dirigirnos al mundo.

- Tristeza: La emoción de la tristeza implica una pérdida o desilusión que nos permite retirarnos hacia nosotros mismos. Es de menor energía que las otras. Si no la experimentamos adecuadamente, no podríamos retirarnos para luego seguir buscando en otro lugar la satisfacción de nuestras necesidades.

- Enojo: El enojo permite defenderse del ambiente y poner límites, pues capacita a las personas de lo que les hace bien y lo que no. Si no se experimenta adecuadamente, se puede ser presa de los otros con facilidad. Basañez (2016) indica que los cambios físicos que provoca enojarse son fruncir el ceño, tensar los músculos, enrojecimiento del rostro, respiración acelerada e incremento de la presión sanguínea. El enojo es favorable porque nos ayuda a adaptarnos. De hecho, ocultarlo o intentar evitarlo generaría más problemas. El reto es darle la intensidad adecuada para expresarlo, y va desde una molestia ligera hasta una ira descontrolada (Basañez, 2016), que puede volverse negativa si es frecuente, prolongada y desproporcionada.

- Alegría: La emoción de la alegría es fundamental para mantenernos vivos y con energía. Es experimentada cuando se satisface alguna necesidad en el presente inmediato. Quien no la vivencia plenamente le ocasiona una falta de vitalidad. La alegría despierta excitación e interés, lo cual nos lleva a explorar nuevos horizontes. De hecho, nos ha ayudado a evolucionar y a adaptarnos a los distintos lugares donde hemos tenido que vivir.

En síntesis, al facilitar los procesos emocionales generalmente no se está trabajando directamente con la emoción, ya que esta es efímera, de ahí que no ocurra frente a nuestros ojos. Esto significa que en realidad se trabaja con lo que queda después la emoción, es decir, el sentimiento. 


\section{Revista Iberoamericana de las Ciencias de la Salud}

\section{Sentimiento}

El sentimiento es la elaboración y representación cognitiva de experiencias como el estado del cuerpo, la emoción, las percepciones, los recuerdos y los pensamientos del estado del cuerpo en un proceso reactivo. Muñoz (2016) explica que no todo sentimiento proviene de una emoción, sino únicamente de los estados del cuerpo, de las sensaciones. Para tener sentimientos hemos de ser capaces de tener sensaciones y representarlas cognitivamente. Entonces, el sentimiento es una sensación corporal que interpreto y concretizo de acuerdo con mi historia, experiencias propias o también todas aquellas que he introyectado. Mi marco cultural. Las características más importantes son las siguientes:

- Implican una elaboración y representación cognitiva.

- Ocurren posteriormente a las sensaciones y emociones en el proceso emocional.

- Son la parte del sentir que se hace privada, es decir, no pueden ser fáciles de observar por otros, pues son invisibles a todos los que no sean su propio dueño.

- Contribuyen a la regulación de la vida, pero están más al servicio del desarrollo que de la supervivencia.

- El sentimiento suele ser poco intenso, pero es más prolongado que la emoción.

- Si la intensidad del sentimiento perturba la homeostasis del organismo, entonces ha llegado a un límite, que es igual a enfermedad o muerte.

\section{Bienestar subjetivo}

La psicología representa la percepción subjetiva de la calidad de vida en el concepto bienestar subjetivo (BS), el cual expresa la satisfacción de las personas y su grado de complacencia con aspectos específicos o globales de su existencia en el que prevalecen los estados de ánimo positivos. En términos específicos, el BS estaría compuesto por un componente cognitivo que corresponde a la satisfacción con la vida, ya sea de forma general o específica; y el componente afectivo, que se refiere a la presencia de sentimientos positivos, denominados felicidad. La satisfacción con la vida y el componente afectivo del bienestar subjetivo parece correlacionarse, ya que ambos se encuentran influidos por la valoración que realiza la persona acerca de los sucesos, actividades y circunstancias en las que se desarrolla su vida (Denegri, García y González, 2015). 


\section{Revista Iberoamericana de las Ciencias de la Salud}

Ojeda-García (2011) comparte la aportación del doctor Myhaly Csikszentmihalyi, quien señala que la felicidad es el sentimiento más anhelado por todos. Las percepciones sobre la vida de cada uno son el resultado de muchas fuerzas que conforman la propia experiencia de su significado e interpretación. Cada una produce un impacto que hace sentir bien o mal a la persona. Esta sensación se manifiesta con la intensidad y nivel de alegría de vivir, lo que es traducido como bienestar subjetivo. El BS es vital para desempeñarse en cualquier ámbito (social, laboral, familiar, individual, etc.), por lo que cada persona debe prepararlo, cultivarlo, buscarlo y alimentarlo cotidianamente. Por eso, se puede decir que la felicidad y el bienestar son inseparables, de ahí que con el incremento en el nivel de apreciación de felicidad y bienestar subjetivo la persona se vuelva más creativa, logre un mayor contacto y relaciones más profundas con otras personas, expanda su lenguaje y, en definitiva, su cuerpo y sus expresiones se vean más radiantes (Ojeda-García, 2011).

En este sentido, Pontaza (26 de octubre de 2017) considera que hay pacientes que responden de forma provechosa, pues después del cáncer empiezan a valorar la vida de manera distinta. Esto tiene que ver también con si se le dio un seguimiento psicológico durante el proceso, cómo estaba la paciente en este y cómo fue adaptándose a todos los cambios experimentados.

La mayor parte de los estudios realizados hasta ahora se interesan por la influencia de los diferentes estilos de apego en el afrontamiento de la enfermedad, y se basan en la idea de que el diagnóstico del cáncer activa las conductas de apego. Si algo caracteriza al cáncer es la sensación de amenaza. Por eso, la calidad del vínculo establecido entre la persona enferma y sus cuidadores debe ser particularmente relevante para su bienestar emocional.

Se han realizado indagaciones sobre las variables de sensibilidad a los cambios vitales que acompañan al diagnóstico y al tratamiento del cáncer de mama, y se ha explorado su valor predictivo sobre el bienestar, entendido como la presencia de afectos positivos y/o la ausencia de afectos negativos. Los resultados indican que el componente temor al rechazo y al abandono del vínculo de apego está fuertemente relacionado con el bienestar, como también lo está la presencia de contrariedades cotidianas y la presencia de síntomas relacionados con la enfermedad y el tratamiento. Como era esperable, tanto la capacidad funcional de las pacientes como la gravedad de los síntomas que perciben correlacionan fuertemente con los medidores del estado de ánimo. Cuanto más alto es el nivel funcional y más baja la presencia de síntomas, más alto es el afecto positivo y más bajo el negativo. 
Cuanto más temor tienen las pacientes a ser abandonadas o rechazadas en sus relaciones interpersonales, más informan de altas emociones negativas y de bajas emociones positivas.

El cáncer, por tanto, implica dos tipos de cambios vitales. Por un lado, pone a pacientes y a allegados ante la perspectiva de la muerte. Por otro, las intervenciones médicas y los fuertes y largos tratamientos suponen una grave perturbación de la vida cotidiana. Desde la psicología del desarrollo, la vida puede definirse como una sucesión de tareas encaminadas a alcanzar ciertas metas (ya sean cotidianas o perentorias) y a la construcción de las habilidades que se necesitan para ello (Alonso, Fontanil y Ezama, 2016).

\section{Psicoterapia corporal}

Todas las definiciones coinciden en que en esta corriente se "corporaliza" la vida mental, es decir, se buscan equivalencias, interrelaciones o interfaces entre el ámbito tradicionalmente considerado psicológico y el corporal (Ortiz Lachica, 2016). Este mismo autor cita de la Asociación Europea de Psicoterapia Corporal (EABP) una definición mucho más incluyente, es decir, es una teoría que explicita acerca del funcionamiento del cuerpomente tomando en cuenta la complejidad y las interacciones entre ambos. Su premisa básica es que el cuerpo refleja a la persona como un todo, y hay una unidad funcional entre el cuerpo y la mente (ninguna de las partes domina a la otra). Utiliza una gran variedad de técnicas, algunas de las cuales se usan en o con el cuerpo, e involucran el contacto físico, el movimiento y la respiración.

Hay que pensar que en la práctica se pueden utilizar diferentes combinaciones de factores comunes para otras psicoterapias, como por ejemplo movimiento y postura, respiración, contacto físico, y reconstrucción de eventos significativos. A través de estas se obtiene información que ayuda al diagnóstico, pues proporciona datos acerca de cómo son y de cómo está la persona. También se accede al material psicológico, el cual sirve para conocer y modificar los recuerdos, pensamientos, emociones y sensaciones corporales que se utilizan con el fin de desbloquear el cuerpo o reconstruir un evento significativo.

Aunque en esta investigación el trabajo con los pacientes tiene su base en las técnicas corporales, se considera importante hacer mención de las técnicas gestálticas, ya que van muy de la mano con ello. En esta investigación — como se verá dentro de la intervenciónlas técnicas más utilizadas fueron las expresivas. 
La Gestalt hace una clasificación de técnicas utilizadas en el proceso de la psicoterapia. Se dividen en tres grupos interdependientes entre sí, que a continuación se definirán como supresivas. Estas sirven para descubrir la experiencia oculta detrás de determinada actividad. Fritz Perls argumentaba que en la psicoterapia Gestalt se debía evitar la intelectualización para poner atención y énfasis en la experimentación a través de las técnicas vivenciales. Decía que hablar del problema favorecía la racionalización en lugar de incrementar la toma de conciencia (Salama, 2012). Estas técnicas son experimentar el vacío, no hablar acerca de..., descubrir los debeísmos, saber formular preguntas y respuestas, captar cuando se pide aprobación, y darse cuenta de cuándo se está demandando o exigiendo.

Expresivas: El objetivo es reunir energía suficiente para fortalecer el contenido del darse cuenta del consultante, ya sea sugiriendo la intensificación de la atención o exagerando la acción deliberadamente. Algunas son expresar lo no expresado, terminar o completar la expresión, buscar la dirección y hacer la expresión directa.

Integrativas: El objetivo de esta técnica es integrar las partes alienadas del individuo. Estas son encuentro intrapersonal, asimilación de lo proyectado, fantasías guiadas. La tarea es abordar la conciencia y utilizarlas en el momento oportuno de la sesión, según las necesidades del paciente.

\section{Método}

La presente investigación se realizó con base en una metodología cualitativa, que se caracteriza por presentar los datos producidos desde la perspectiva del otro; es decir, toma las propias palabras de las personas, habladas o escritas, así como su conducta observable (Taylor y Bogdan, 1987). Además, con apoyo del enfoque fenomenológico, se procuró comprender lo subjetivo del pensamiento a partir de la exposición de las experiencias originales de la persona en su contexto (Soto y Vargas, 2017).

La intervención se realizó a partir de una serie de técnicas vivenciales, y se presenta como un estudio de caso. Las técnicas aplicadas fueron la entrevista a profundidad (efectuada en dos sesiones de aproximadamente una hora), donde se exploró la historia de la participante en relación con el desarrollo de su enfermedad y la forma como afectó el resto de su vida; y la narrativa, que se tomó de los relatos y discursos de la participante durante la realización de las técnicas vivenciales que se llevaron a cabo en ocho sesiones de dos horas. Tanto la intervención como la entrevista se llevaron a cabo en un consultorio psicológico al cual la 
participante se presentó voluntariamente. La participante es originaria del municipio de Cuitzeo, Michoacán, México. Tiene 45 años de edad y su estado civil actual es unión libre. Concluyó sus estudios de secundaria y tiene dos hijas.

\section{Resultados}

A partir de las entrevistas y la narrativa de la participante, se sistematizó la información en tres categorías de análisis: los sentimientos que expresa la participante sobre su enfermedad, el manejo que da a sus emociones, y evaluar el desarrollo del bienestar subjetivo de la participante.

\section{Categoría 1. Sentimientos presentes respecto a la enfermedad}

Los sentimientos que se presentaron en la paciente respecto a su enfermedad fueron el miedo, el enojo y la tristeza. Se iniciará analizando el miedo. Este fue originado por cuatro circunstancias diferentes: morir y dejar solas a sus hijas, que su pareja la abandonara por la enfermedad, que le amputen el seno, y las quimioterapias. La palabra "cáncer" la relaciona con muerte. Ella no quiere morir, pues dejaría solas a sus hijas, las cuales sufrirían. El miedo a ser abandonada se sustenta en una experiencia del pasado, cuando una expareja la dejó y le dijo que era fea y que nadie la iba a querer. En consecuencia, se ha creado en ella la idea de que cuando se le caiga el cabello ya no será atractiva para su pareja actual. Por eso, surge el miedo a las quimioterapias. Si le amputan el seno, para ella significaría convertirse en una mujer incompleta, por lo que no va a querer que la vean así.

\section{P. Psi. ¿Por qué piensas que tu pareja te va a dejar?}

Paciente: Él no me dice que me va a dejar; él siempre me demuestra cariño, me dice que le eche ganas y que me opere, que él me va a querer igual. Pero tengo miedo de que mi pareja me deje porque ya no sea una mujer bonita y completa si me quitan mi pecho.

P. Psi. ¿Y si te deja qué pasa?

Paciente: Me quedaré sola nuevamente con mis hijas y con mi enfermedad, y mis hijas sufrirían de nuevo.

P. Psi: Entonces mejor no me opero para que no me deje y que el cáncer avance (se observa que la paciente entra en el darse cuenta 
mediante su expresión no verbal, y bajo un principio de realidad que se refiere a que ella se da cuenta de que tiene ese miedo y por qué lo tiene; comienza a ver que si no se opera, quien abandonará a su pareja y a sus hijas será ella).

Paciente: No me quiero morir (se invita a que exprese y al hacerlo se escuche), no quiero este miedo. Déjame, no me sirves, miedo. Quiero vencer el cáncer...

P. Psi: (una vez expresado todo por lo cual tiene miedo, se le invita a que repita lo siguiente) $Y$ cuando lo digo me siento... (la paciente menciona) bien, tranquila, liberada, relajada. ¿De qué te das cuenta?, ¿qué integras de este momento?

Paciente: Me doy cuenta de que en realidad no me quería operar por miedo a que mi pareja me deje, y que esto lo viví en el pasado y nunca lo dije. También me doy cuenta de que ya no estoy con mi expareja, que ahora estoy con un hombre diferente.

P. Psi: ¿Qué te gustaría decirle a tu pareja ahora?

Paciente: Te pido perdón (refiriéndose a su pareja a través de la técnica) por no aceptar tus cuidados. Hoy me doy cuenta de que estás conmigo y que me quieres, que mi miedo es por lo que yo viví en mi pasado. Este miedo no me va a paralizar. Quiero estar bien y vencer el cáncer.

P. Psi: Reconoce que ahora tu pareja está contigo acompañándote y apoyándote en estos momentos y no te ha abandonado. Acéptalo y recíbelo. ¿Con qué más cuentas para continuar con este proceso?

Paciente: Me tengo a mí misma, y como dijo el doctor: yo soy una parte importante en este proceso, y si yo fallo, no se logrará el objetivo, que es vencer el cáncer. Tengo a Dios.

Por otro lado, otro sentimiento presente en la participante respecto a su enfermedad fue el enojo, el cual posiblemente se relacione con un reclamo a la vida, ya que ella dice: "Yo NO me merezco esta enfermedad porque ya he sufrido mucho y me siento enojada porque yo soy una mujer buena”. Se le preguntó: ¿qué sientes hacia el cáncer, qué le quieres decir, qué 
quieres hacer con él? La paciente comienza a tener contacto con la emoción y expresa lo siguiente:

Paciente: Estoy enojada porque siento que nadie me entiende, porque solo me dicen que le eche ganas.

P. Psi: Entiendo que te sientas así porque no es fácil tener una enfermedad como esta. Ahora te pido que pongas delante de ti al cáncer, aquí está frente a ti. ¿Por qué no mereces estar enferma?

Paciente: Porque soy una mujer buena

P. Psi: Entonces, ¿porque eres una mujer buena no te puedes enfermar?

Paciente: No, porque yo he sufrido mucho, y esta enfermedad es muy agresiva que me está haciendo sufrir más, y me siento enojada con Dios, con mi familia, con todas las personas que me dicen que le eche ganas, porque ellas no están enfermas, no tienen cáncer (comienza a llorar).

Se le proporciona periódico y se le dice: P. Psi: Aquí tienes el cáncer en tus manos (representación simbólica, atendiendo a la necesidad del momento de la paciente). ¿Qué le quieres decir?

Paciente: Me siento enojada porque apareciste en mi vida (se le pide que exprese todo lo que le quiere decir). ¡No te quiero en mi cuerpo! ¡Vete! ¡Me estás haciendo daño! ¡No te quiero!

P. Psi: ¿Qué quieres hacer con él?

Paciente: Romperlo, deshacerme de él (se le pide que continúe expresando). Salte de mi cuerpo, quiero acabar contigo, destruirte y lo voy hacer (se le dice que repita "Y lo estoy haciendo, te estoy destruyendo").

P. Psi: Entonces destrúyelo, acaba con él (la paciente rompe el periódico y al mismo tiempo expresa todo lo que le ha causado a su vida. Después de varios minutos comienza a descender su energía y va terminando de expresar y de romper el periódico). 
Para finalizar esta primera categoría, se explicará el último sentimiento presente en la participante, la tristeza respecto a la enfermedad. La paciente comparte su sentir cuando le dieron el diagnóstico médico. "Al principio cuando me diagnosticaron, yo no salía, me sentía triste, sin ganas de salir, de repente sentía que se me bajó toda mi autoestima, pero me hacía la fuerte". Esta tristeza le impedía salir de su cuarto. Menciona que lloraba a solas y a escondidas para que sus hijas no la vieran sufrir.

\section{Categoría 2. Manejo que la participante da a sus emociones}

En esta categoría se pretende analizar la manera en la que la participante maneja sus emociones y las consecuencias que esto genera en su salud. En el transcurso de la intervención se observó que la participante utiliza como mecanismo de defensa la represión. De hecho, a lo largo de su vida no ha expresado sus emociones, aunque comentó situaciones pasadas muy difíciles. Reprimir determinadas emociones puede ser saludable, a corto plazo, pues pude darle a la persona un poco más de tiempo para enfrentar otras que la pudieran sobrepasar si actuara súbitamente. Sin embargo, no es saludable negarlas indefinidamente.

A lo largo de las sesiones trabajadas, ella mencionó: "Al principio sentía miedo por la enfermedad, pero ahora me doy cuenta que ese miedo tiene que ver con que mi pareja me deje". La intervención le dio la oportunidad para hablar de los sentimientos respecto a su enfermedad, para expresarlos e identificarlos, y aunque al principio no le era sencillo, pareciera que hubo un cambio. De hecho, en la tercera sesión su rostro se veía cargado de energía y vitalidad. Ella afirmó que estar asistiendo a las sesiones le hacía sentirse diferente.

Expresar sus sentimientos ahora la mantiene tranquila y, en su momento, le ayudó para enfrentar su enfermedad, lo cual la llevó a tener una mejor adhesión al tratamiento con quimioterapia. La participante indicó que utilizó las técnicas de respiración aprendidas en la intervención para estar tranquila y disminuir el miedo.

\section{Categoría 3. Desarrollo de bienestar subjetivo}

En esta categoría se describe cómo la expresión de sentimientos de la participante fortaleció el desarrollo de su bienestar subjetivo. Se divide en tres subcategorías.

1) Sentimientos respecto a su enfermedad después de la intervención: Trascendidos el miedo, el enojo y la tristeza, ahora puede sentir gratitud por su enfermedad. Aprendió a valorar a su familia y a la vida. Ella menciona: "Tengo el tiempo suficiente para estar con mi 
familia, pongo de mi parte en los procesos médicos, y aunque me sienta mal con las quimios, porque estoy acostada mucho tiempo en mi casa, sé que estoy con ellos. Tengo el tiempo para enseñarles a mis hijas y estar con ellas". El cáncer para su vida ahora lo siente como una enfermedad no grave, ya no la siente pesada. Antes, el cáncer le daba miedo; sin embargo, esta intervención le ayudó a verlo diferente, como una enfermedad que, siendo atendida, se puede superar.

Se trabajó a través de una fantasía guiada. Se le pidió que colocara la mano en su seno afectado para agradecerle por lo que le ha dejado y enseñado. Ella dijo: "Te doy las gracias porque me has hecho más fuerte, porque me has acercado más a mi familia. Gracias también porque conocí a las psicólogas". Agradece también al seno que le van a retirar. "Gracias por el tiempo que estuviste conmigo, porque me diste la dicha de ser mujer y de alimentar a mis hijas. Gracias por hacerme ver bonita".

$\mathrm{Al}$ expresar sus emociones, se siente agradecida y dice: "Me he dado cuenta que tengo a un gran hombre a mi lado y he decidido dedicarme más a disfrutar el tiempo con mi familia".

2) Reconocimiento de recursos, habilidades y fortalezas: La participante habla sobre tener muchas ganas de vivir porque tiene toda una vida por delante, rodeada de gente que la ama, y aunque tenga esta enfermedad, ella se siente capaz de superarla. Menciona que es una mujer fuerte, valiente y bonita, y que aun sin tener un pecho, vale igual o mucho más. Se encuentra tranquila, relajada y contenta. No le teme a la enfermedad. "Yo puedo superar el cáncer y dar mucho más de lo que ya tengo".

3) Adhesión al tratamiento: Recibir la noticia de una enfermedad terminal anticipa dos opciones: la primera, abandonar totalmente cualquier ayuda de tipo médico que se pueda dar; y la segunda, seguir las indicaciones del equipo médico, ya sea para atacar a la enfermedad directamente o para tratarla, haciéndola más soportable. En tal sentido, la participante de esta investigación, quien ha decidido continuar con el tratamiento para vencer esta enfermedad, se muestra tranquila y con planes para el futuro. No piensa renunciar a sus metas. Gracias a todo el trabajo personal que ha logrado durante las intervenciones, comenta:

"Me ha ayudado para seguir mis metas tanto individuales como familiares, ya que quiero seguir estudiando y aprendiendo más. También queremos ampliar la casa, quiero trabajar y apoyar a mi esposo. En cuanto a mi tratamiento no he tenido dudas y me he sentido apoyada por los doctores y por las psicólogas. No olvido el primer día que fui a la cita al hospital; ese 


\section{Revista Iberoamericana de las Ciencias de la Salud}

día el doctor fue muy amable y me explicó que son tres cosas muy importantes que se deben de cumplir en este proceso: yo, ellos y mi familia, y que si una de estas tres partes fallaba, todo se complicaría. Desde ese momento entendí que todo era por mi salud y por mi vida. Soy la parte más esencial en este proceso y empecé a echarle todas las ganas para luchar contra esta enfermedad. Aprendí a ponerle atención mi cuerpo, todas las sensaciones y emociones que siento trato de expresarlas, me permito preguntar más a los doctores cosas que no me quedan claras. Ahora entiendo que mi cuerpo es único: siente y expresa, y junto con él he aprendido a escuchar y a ponerle atención a mi cuerpo, y eso me ha ayudado en mi tratamiento".

La participante ha escuchado atentamente las indicaciones médicas y psicológicas. Con una actitud de compromiso, perseverancia, independencia, armonía y amor, la participante continúa acudiendo a sus citas posquirúrgicas, y aun habiendo terminado las quimioterapias, sigue con sus revisiones. Progresivamente va volviendo al reajuste de su vida, adaptándose y enfrentando los cambios de este hecho inesperado.

\section{Discusión}

En todos los procesos de la vida hay energía (en el movimiento, en el sentimiento y en el pensamiento). De hecho, todos pueden interrumpirse si falta energía al organismo. En tal sentido, para que una persona recupere la energía se utiliza de manera inmediata el aumento de su toma de oxígeno, de ahí que se le pida que respire más profunda y plenamente.

Ahora bien, no se puede hablar del concepto carga de energía sin considerar lo que es una descarga de energía. Los organismos vivos solo son capaces de funcionar cuando existe equilibrio entre la carga y la descarga de su energía. Mantienen un nivel de energía adecuado a sus necesidades y oportunidades. El placer y la satisfacción constituyen la experiencia inmediata de las actividades de la autoexpresión. Limítese el derecho de una persona para expresarse y se habrán disminuido las oportunidades que tiene de gozar del pacer y del vivir creativo. En otras palabras, no puede elevarse el nivel de energía personal solamente por medio de la respiración. Tienen que abrirse los canales de autoexpresión por medio del movimiento, la voz y los ojos para que pueda generarse una mayor descarga. Por ello, se combinan la respiración, el sentimiento y el movimiento junto con el intento de relacionar el funcionamiento actual energético del individuo con la historia de su vida. Así 
se descubren paulatinamente las fuerzas interiores (los conflictos) que impiden que la persona pueda funcionar a todo su potencial energético. Cada vez que se resuelve el conflicto interno, sube el nivel individual de energía; en pocas palabras, la persona carga y descarga más energía en actividades creadoras, placenteras y satisfactorias (Lowen, 2018).

En el apartado de resultados se explicó que los sentimientos presentes en la participante respecto a su enfermedad fueron el miedo, el enojo y la tristeza. El origen del miedo inicia en la falta de satisfacción de la necesidad de seguridad y la falta de confianza. Su principal función es la protección, y predispone a la huida. Es importante en la vida de los seres humanos para cuidar de sí mismos ante los peligros constantes (Muñoz, 2016).

El impacto que causa la mastectomía en la mujer genera diferentes emociones, y la intensidad con la que se presenten va a estar mediada por los estilos de afrontamiento con los que se cuenten. Se encontró que ante la cirugía las mujeres presentan sentimientos de pérdida, mutilación, desvalorización de la imagen corporal, frustración, tristeza, enojo, y trastornos de ansiedad y depresión. El impacto del cáncer y sus tratamientos oncológicos limitan la vida social, familiar y personal de las pacientes, de ahí la importancia de intervenir en estas problemáticas con el fin de lograr un reconocimiento de la nueva imagen corporal y, por ende, mejorar la calidad de vida.

Es importante el apoyo psicooncológico para que las pacientes aprendan nuevas habilidades de autorregulación emocional que les permitan disminuir los efectos de estos cambios corporales. Estas estrategias le brindan a la paciente la oportunidad de conocer otro tipo de recursos para sobrellevar los efectos de la cirugía. De hecho, mantenerlas funcionales y participativas durante su tratamiento permite que posteriormente puedan tomar decisiones sobre intervenciones (como la reconstrucción mamaria) que las lleven a sentirse mejor (Martínez-Basurto, Lozano-Arrazola, RodríguezVelázquez, Galindo y Alvarado, 2014).

Muñoz (2016) explica que la etiología de la tristeza se basa en la vivencia frecuente de pérdidas y decepciones. Esta tiene que ver con una sensación de desilusión que nos permite retirarnos hacia lo que consideramos mejor para nosotros, y se caracteriza por una falta de energía. Si no fuere experimentada esta emoción adecuadamente, no podríamos retirarnos de ese hecho doloroso para después seguir buscando la satisfacción de nuestras necesidades en otro escenario. 


\section{Revista Iberoamericana de las Ciencias de la Salud}

Villa, Font y Caba (2016) encontraron algo semejante a esta investigación, pues observaron que las mujeres que acababan de recibir un diagnostico experimentaban rabia, miedo y nerviosismo de forma ligeramente más intensa que las pacientes de otras situaciones médicas. Se ha encontrado que el malestar es más intenso durante la fase de diagnóstico; sin embargo, la calidad de vida de las mujeres con cáncer de mama mejora a medida que trascurre el tiempo desde el diagnóstico hasta el tratamiento. En todo caso, parece ser que la fase de diagnóstico es la que suele generar mayor malestar emocional, como se ha constatado en otros estudios. En relación con el cambio emocional favorable, este era mayor en aquellas mujeres que se encontraban en la fase de tratamiento o en la fase de seguimiento (intervalo libre), y en aquellas mujeres que ya habían realizado algunas visitas con la psicooncóloga previamente. Esto último indicaría que se da un cierto efecto de aprendizaje terapéutico.

De igual manera, se considera que cuando más experimentamos emociones y no las expresamos, tanta más presión vamos juntando dentro, y nuestra mente percibe que estamos en situación de peligro, con lo cual sentimos que debemos huir (encerrar aún más nuestras emociones) o pelear (enojarnos contra las emociones) (Colbert, citado por Atencio y Ramírez, 2019).

Los pacientes atraviesan varias fases en el proceso de asumir que padecen una enfermedad y de aprender a vivir con ella. Algunos se sienten vulnerables, confundidos y preocupados por su salud y su futuro. Estos sentimientos forman parte del proceso de afrontamiento. En este proceso, el apoyo familiar que recibe el paciente es un aspecto fundamental, ya que la familia es la mayor fuente de apoyo social y personal que pueden disponer las personas. La importancia del apoyo familiar se hace visible en este relato biográfico, temática a la que la informante dedica un mayor número de palabras (Correa, 2017).

El apoyo de redes sociales, vínculo laboral, calidad de las relaciones familiares, rasgos de personalidad y estilo de afrontamiento juegan un papel significativo en el proceso de enfermedad. Las estrategias de afrontamiento son entendidas como un conjunto de esfuerzos tanto cognitivos como comportamentales que cambian constantemente y se desarrollan para manejar las demandas externas e internas, que son evaluadas como excedentes y desbordantes de los recursos del individuo. Cada individuo presenta diferentes formas relativamente estables de afrontar cada situación (Cerquera, Matajira y Vásquez, 2017). 


\section{Revista Iberoamericana de las Ciencias de la Salud}

Siguiendo a estos autores, cabe destacar que el estilo de afrontamiento que la paciente de esta investigación tuvo para enfrentar su enfermedad fue activo, pues se trata de personas que movilizan sus esfuerzos para los diferentes tipos de solución ante la situación.

Al llevarse a cabo la intervención se pretendía que la paciente desarrollara una adherencia al tratamiento con la finalidad de promover su bienestar subjetivo. La psicología de la salud ha utilizado el término adherencia terapéutica, entendida como una implicación activa y voluntaria del paciente en el curso de un comportamiento aceptado de mutuo acuerdo con su médico con el fin de producir un resultado terapéutico deseado (Martín, Grau y Espinosa, 2014).

Se considera que la participante de esta investigación, al final de todo el proceso de intervención psicoterapéutica, logró trascender su enfermedad y promover en su vida el desarrollo de bienestar subjetivo. La felicidad es un estado de tipo emocional y cognitivo de las personas que se define como el predominio de la frecuencia de ocurrencia de experiencias emocionales positivas sobre las negativas, implicando la capacidad de amar, trabajar, relacionarse socialmente y controlar el medio (Denegri, García y González, 2015).

\section{Conclusiones}

Después de haber realizado satisfactoriamente esta investigación, se considera que a una persona con una enfermedad crónica se le despiertan emociones causadas por la enfermedad y por el pasado, que no fueron expresadas en su momento. En este sentido, el malestar emocional es una experiencia desagradable, multifactorial, de naturaleza psicológica, social y/o espiritual que interfiere con la habilidad de afrontar de manera efectiva el cáncer, sus síntomas físicos y su tratamiento. Las emociones presentadas en la participante por una enfermedad etiquetada como mortal y por las vividas en el pasado fueron el miedo, el enojo y la tristeza. Se observó que a la paciente le costó trabajo expresar sus sentimientos, y utilizó como mecanismo de defensa la represión.

Se encontró también que al llevar a la paciente a expresar sus sentimientos respecto a su enfermedad promovió su bienestar subjetivo; esto a través de técnicas psicocorporales, pues por medio de su utilización se activa la energía de la persona para tomar contacto con su cuerpo, lo que a su vez revive emociones profundas no expresadas. Esto genera alivio del dolor emocional, donde los sentimientos se convierten ahora en una construcción y desarrollo 
de su potencial como persona. Son sentimientos proporcionados, es decir, su frecuencia va de acuerdo a las circunstancias vividas.

Cabe mencionar que el rostro de la paciente cambió al expresar cómo se sentía respecto a su enfermedad. En otras palabras, su cara y cuerpo irradiaban tranquilidad y vida. Gracias a esta investigación y a través de la intervención, con la utilización de las técnicas psicocorporales, analizando el manejo de las emociones y su expresión, se logró promover el bienestar subjetivo en la paciente. Esto sirvió para que la participante decidiera formar parte del programa de tratamiento y atendiera las indicaciones médicas.

En cuanto a la psicoterapia corporal, se trabajó con esta porque su premisa básica dice que el cuerpo refleja a la persona como un todo, y que hay una unidad funcional entre el cuerpo y la mente. Se utilizó esta ciencia porque sus técnicas involucran contacto físico, así como movimiento y respiración. Esto ayudó a que la participante tuviera un fluir en la energía corporal, lo que impactó en el contacto con las emociones. De este modo se cumplieron los objetivos de la terapia corporal en cuanto a propiciar la ampliación de la consciencia, la expresión de las emociones, el alivio al sufrimiento, la capacidad para sentir placer y, en general, el crecimiento y desarrollo personal.

Ya desde el psicoanálisis — con varias figuras representativas como Freud o Reich, este último considerado como el padre de la psicoterapia corporal — se ha trabajado con el cuerpo y la mente, pues mientras el consultante narraba su historia, con posturas corporales y la respiración se lograban contactar las emociones para ser expresadas. Por eso, consideramos que el trabajo con la psicoterapia corporal abrirá la oportunidad para que más mujeres diagnosticadas con cáncer puedan expresar sus sentimientos respecto a la enfermedad. Así podrán tomar conciencia sobre el valor que como mujeres poseen y sobre las opciones para combatir la enfermedad apegadas a un tratamiento a tiempo.

En definitiva, se propone continuar desarrollando programas, ya sean cursos o talleres de psicoterapia corporal, para pacientes con cáncer y con enfermedades crónicas degenerativas, pues de esta manera se puede promover la expresión de sentimientos dentro de un clima de empatía, respeto, confidencialidad, acompañamiento y ética profesional. En caso contrario, reprimir las emociones puede generar en los pacientes un estado de frustración y malestar que deteriorará aún más su salud. En tal sentido, vale recalcar el apoyo y acompañamiento de la familia, el trabajo del equipo multidisciplinario del área de salud y el empeño del paciente como factores claves para el avance y recuperación física y psicológica 
del enfermo. Con esos programas propuestos se intentará ayudar al paciente a transformar su percepción negativa de la enfermedad por otra que le permita vislumbrar la oportunidad para desarrollar su potencial humano.

\section{Futuras líneas de investigación}

Como en esta investigación se atendió a una sola persona, se podría incluir en el futuro el estudio de los beneficios del trabajo grupal. También sería interesante investigar el bienestar subjetivo de personas con distintas enfermedades crónico-degenerativas con la finalidad de analizar si los pacientes manejan las mismas emociones. De igual manera, se considera relevante revisar si existen cambios en los sentimientos que manifiestan durante las distintas fases de su enfermedad.

\section{Referencias}

Alonso, Y., Fontanil, Y. y Ezama, E. (2016). Apego y bienestar en mujeres en proceso de tratamiento de cáncer de mama. Anales de Psicología, 32(1), https://scielo.isciii.es/scielo.php?script=sci_arttext\&pid=S021297282016000100004

Atencio, E. y Ramírez, R. (2019). Una mirada reflexiva al modelo escuela nueva de la institución educativa Guaimaral (sede Altomira) (tesis de maestría). Colombia: Universidad de la Costa. Recuperado de https://repositorio.cuc.edu.co/bitstream/handle/11323/5695/Una\%20mirada\%20refl exiva\%20al\%20modelo\%20escuela\%20nueva\%20de\%20la\%20instituci\%C3\%B3n $\% 20$ educativa\%20Guaimaral\%20(Sede\%20Altomira).pdf?sequence=1

Basañez, L. (2016). Y tus emociones, ¿qué dicen? Aprende a manejarlas. México: Pax.

Cerquera, A., Matajira, Y. y Vásquez, E. (2017). Estrategias de afrontamiento en pacientes diagnosticados con cáncer de mama y el papel del psicólogo. Psicología GEPU, 8(2), 144-154. Recuperado de https://revistadepsicologiagepu.es.tl/Estrategias-deafrontamiento-en-pacientes-diagnosticados-con-c\%E1ncer-de-mama-y-el-papel-delpsic\%F3logo.htm 
Correa, M. (2017). Impacto psicológico frente al diagnóstico de cáncer de mama. Primeras reacciones emocionales. Index de Enfermería, 26(4). Recuperado de https://scielo.isciii.es/scielo.php?script=sci_arttext\&pid=S113212962017000300015

Denegri, M., García, C. y González, N. (2015). Definición de bienestar subjetivo en adultos jóvenes profesionales chilenos. Un estudio con redes semánticas naturales. CES Psicología, $\quad 8(1) 7 . \quad 7-97 . \quad$ Recuperado de https://www.redalyc.org/pdf/4235/423539425006.pdf

Duque, H. y Vieco, P. (2012). Conozca sus emociones y sentimientos. Talleres vivenciales. Bogotá: San Pablo.

Lowen, A. (2018). Bioenergetica. México: Diana.

Martín, L. D., Grau, J. A. y Espinosa, A. D. (2014). Marco conceptual para la evaluación y mejora de la adherencia a los tratamientos médicos en enfermedades crónicas. Revista Cubana de Salud Pública, 40(2). Recuperado de https://www.redalyc.org/articulo.oa?id=21431239007

Martínez-Basurto, A., Lozano-Arrazola, A., Rodríguez-Velázquez, A., Galindo, Ó. y Alvarado, S. (2014). Impacto psicológico del cáncer de mama y la mastectomía. Gaceta Mexicana de Oncología, 13(1), 53-58.

Muñoz, M. (2016). Emociones, sentimientos y necesidades. Una aproximación humanista. México: Araucaria.

Ojeda-García, A. (2011). El bienestar subjetivo como resultado de la apreciación. ¿Qué tan felices somos? Psicología Iberoamericana, 19(2), 5-8. Recuperado de https://www.redalyc.org/pdf/1339/133921440001.pdf

Ortiz Lachica, F. (2016). Psicoterapia corporal. Bases teóricas de la práctica. México: Pax.

Palacios-Espinosa, X., González, M. I. y Zani, B. (2015). Las representaciones sociales del cáncer y de la quimioterapia en la familia del paciente oncológico. Avances en Psicología Latinoamericana, 33(3), 497-515. Doi: https://doi.org/10.12804/ap133.03.2015.09

Pallarés, M. (2013). Emociones y sentimientos. Barcelona: Edición Kindle.

Pontaza, D. (26 de octubre de 2017). Psicología: las emociones y el cáncer de mama. TecReview. Recuperado de https://tecreview.tec.mx/2017/10/26/ciencia/psicologialas-emociones-cancer-mama/ 
Ramos Rocha, L. E. (2004). Los sentimientos en psicoterapia Gestalt. En M. M. Polit, una figura de la Gestalt. Guadalajara, Jalisco: Ediciones de la Noche.

Salama, H. (2012). Encuentro con la psicoterapia Gestalt. México: Centro Gestalt. Recuperado https://www.academia.edu/8175272/Gestalt_2_0_Actualizaci\%C3\%B3n_en_Psicot erapia_Gestalt

Soto, C. y Vargas, I. (2017). La fenomenología de Hursserl y Heidegger. Cultura de los Cuidados, 21(48), 43-50. Doi:10.14198/cuid.2017.48.05

Taylor. S. y Bogdan, R. (1987). Introducción a los métodos cualitativos de investigación. Paidós.

Verdugo, J. C., Ponce de León, B. G., Guardado-Llamas, R. E., Meda-Lara, R. M., UribeAlvarado, J. I. y Guzmán-Muñiz, J. (2013). Estilos de afrontamiento del estrés y bienestar subjetivo en adolescentes y adultos jóvenes. Revista Latinoamericana de Ciencias Sociales: Niñez y Juventud, 11(1), 79-91.

Villa, L., Font, A. y Caba, L. (2016). Estado emocional en mujeres con cáncer de mama: variación experimentada después de una sesión psicooncológica basada en el counselling y la psicologia positiva. Psicooncología, 13(2-3), 205-225. Recuperado de https://revistas.ucm.es/index.php/PSIC/article/view/54433 


\begin{tabular}{|c|c|}
\hline Rol de Contribución & Autor(es) \\
\hline Conceptualización & $\begin{array}{l}\text { Ana Karen Osornio García (principal), Belem Medina Pacheco } \\
\text { (igual) }\end{array}$ \\
\hline Metodología & María Inés Gómez del Campo del Paso \\
\hline Software & No aplica \\
\hline Validación & No aplica \\
\hline Análisis Formal & $\begin{array}{l}\text { Ana Karen Osornio García (apoyo), Belem Medina Pacheco } \\
\text { (principal), María Inés Gómez del Campo del Paso (apoyo) }\end{array}$ \\
\hline Investigación & Ana Karen Osornio García \\
\hline Recursos & $\begin{array}{l}\text { Ana Karen Osornio García, Belem Medina Pacheco, María } \\
\text { Inés Gómez del Campo del Paso (igual) }\end{array}$ \\
\hline Curación de datos & $\begin{array}{l}\text { Belem Medina Pacheco, María Inés Gómez del Campo del } \\
\text { Paso (igual) }\end{array}$ \\
\hline $\begin{array}{l}\text { Escritura - Preparación del } \\
\text { borrador original }\end{array}$ & Ana Karen Osornio García \\
\hline $\begin{array}{l}\text { Escritura - Revisión y } \\
\text { edición }\end{array}$ & $\begin{array}{l}\text { Belem Medina Pacheco, María Inés Gómez del Campo del } \\
\text { Paso (igual) }\end{array}$ \\
\hline Visualización & $\begin{array}{l}\text { Belem Medina Pacheco, María Inés Gómez del Campo del } \\
\text { Paso (igual) }\end{array}$ \\
\hline Supervisión & $\begin{array}{l}\text { Belem Medina Pacheco, María Inés Gómez del Campo del } \\
\text { Paso (igual) }\end{array}$ \\
\hline Administración de Proyectos & $\begin{array}{l}\text { Facultad de Psicología, Universidad Michoacana de San } \\
\text { Nicolás de Hidalgo. }\end{array}$ \\
\hline Adquisición de fondos & $\begin{array}{l}\text { Coordinación de Investigación Científica de la Universidad } \\
\text { Michoacana de San Nicolás de Hidalgo. }\end{array}$ \\
\hline
\end{tabular}

